\title{
A continuous non-linear shadowing model of columnar growth
}

\author{
T.H. Vo Thi ${ }^{1}$, J.-L. Rouet ${ }^{1,2}$, P. Brault ${ }^{3} \S$, J.-M. Bauchire ${ }^{3}$, S. \\ Cordier $^{1}$ and C. Josserand ${ }^{4}$ \\ ${ }^{1}$ Laboratoire de Mathématiques et Applications, Physique Mathématique d'Orléans, \\ UMR6628 CNRS-Université d'Orléans BP 6759, 45067 Orléans Cedex 2, France \\ ${ }^{2}$ Institut des Sciences de la Terre d'Orléans UMR6113 CNRS/Université d'Orléans, \\ 1A rue de la Férollerie, 45071 ORLEANS CEDEX 2 \\ ${ }^{3}$ Groupe de Recherches sur l'Energétique des Milieux Ionisés, UMR6606 \\ CNRS-Université d'Orléans BP 6744, 45067 Orléans Cedex 2, France \\ ${ }^{4}$ Institut Jean Le Rond d'Alembert, UMR 7190 CNRS-Paris VI Case 162, UPMC, 4 \\ place Jussieu, 75252 Paris Cedex 05, France \\ E-mail: Jean-Louis.Rouet@univ-orleans.fr, \\ Stephane.Cordier@univ-orleans.fr
}

\begin{abstract}
We propose the first continuous model with long range screening (shadowing) that described columnar growth in one space dimension, as observed in plasma sputter deposition. It is based on a new continuous partial derivative equation with non-linear diffusion and where the shadowing effects apply on all the different processes.
\end{abstract}

PACS numbers: 81.15.Aa, 68.35.Ct, 05.40.-a

Submitted to: J. Phys. D: Appl. Phys. - Fast Track Communications

$\S$ Corresponding author: Fax +33(0)2 384171 54, e-mail: Pascal.Brault@univ-orleans.fr 
Plasma sputtering is a common process for film growth which often exhibits wide columns more or less close packed separated by thin deep grooves [1, 2, 3, 4, This columnar growth mainly results from a shadowing instability [5, 6, 7], where the elevated parts of the surface are more exposed to the sputtering while they shadow the incoming particles to the lower parts. The modelization of this shadowing instability has been well described by probabilistic Monte-Carlo methods (MC) [8, 9, 10] and also with continuous models based on partial derivative equations (PDE) [6, 17, 11, 12, 13, 14, 15, 16] including the seminal work of Bales and Zangwill [四]. However, both approach fail to describe at long times the strongly nonlinear columnar microstructures observed recently (see (2) for instance). In fact, although the continuous models gives tall and well separated columns at early time, only few sharp peaks remain later on [6, 14, 16]. Columnar structure using PDE has already been obtained by Gillet et al. 17 but in that case no shadowing effect was taken into account! On the other hand, discrete approaches using MC methods including shadowing have been developped and showed a fair description of the columnar structure, particularly through the formation of sharp column sides. However, these models cannot avoid the coarsening of the columnar structures showing larger and larger plateau as time increases, in contrast with experimental observations.

The goal of this paper is to present a new continuous non-local model which includes both non-linear shadowing and diffusion effects to simulate columnar-like growth. We consider a two dimensional model where the one dimensional (1D) surface described by $h(x, t)$ is subjected to receive particles from all directions not shadowed by the surface itself. Our starting point is deduced from the models developped initially by Bales and Zangwill[1] and by Karunasiri et al [6]:

$$
\frac{\partial h}{\partial t}=R \Omega(x,\{h\}) \sqrt{1+(\nabla h)^{2}}+\nu \nabla^{2} h+\eta
$$

where the deterministic deposition term $R$ is multiplied by the solid angle $\Omega(x,\{h\})$ which modelizes the shadowing effect as a long range screening (see figure 1). $\nu$ is the diffusion/relaxation coefficient while $\eta$ is the usual noise with zero mean $\langle\eta\rangle=0$ and its correlation given by $<\eta(x, t) \eta\left(x^{\prime}, t^{\prime}\right)>=2 D \delta\left(x, x^{\prime}\right) \delta\left(t, t^{\prime}\right)$.

For small surface angles, we retrieve a KPZ-like equation [18] with shadowing effects (defining $\lambda=\pi R$ ):

$$
\frac{\partial h}{\partial t}=\nu \nabla^{2} h+\frac{\lambda}{2}(\nabla h)^{2}+R \Omega(x,\{h\})+\eta .
$$

A complete study of these equations has shown that it is unable to reproduce columnar shapes corresponding to experiments and MC simulations [7, 14. Indeed, in the most favourable situation, only broad peaks emerge instead of flat columns. Experiments suggest thus that the diffusion should be enhanced in the region more exposed to the flux. Moreover, we will assume that the flux also increases (greater than for normal shadowing) on the top of the columns compared to the grooves. Although we have no strong argument for it, we expect some point effect near the sharp edge 


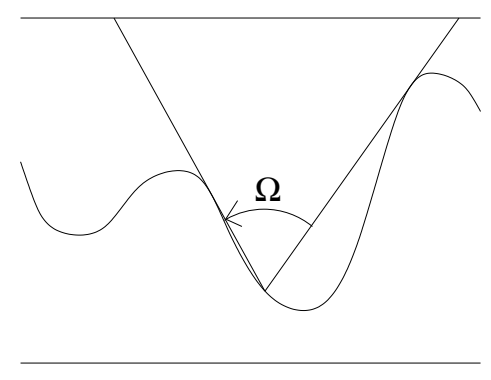

Figure 1. Shadowing process : interface $h(x, t)$ and solid angle $\Omega(x,\{h\})$.

to be responsible of this process. We then propose the following stochastic differential equation where the main ingredients are non-linear shadowing effects and diffusion:

$$
\frac{\partial h}{\partial t}=g(\Omega(x,\{h\}))\left(R \sqrt{1+|\nabla(h)|^{2}}+\nu \nabla^{2} h+\eta\right)
$$

In this equation $g(\Omega)$ is a given function of the solid angle $\Omega$. Therefore, in order to increase the shadowing effect and the diffusion from top to edges and bottom, $g(\Omega)$ has to be stronger than linear, and we will take later on for the numerics $g(\Omega)=\Omega^{2}$ . The fact that this function, which modelizes the shadowing, is in factor to the right hand term will obviously increase the deposit rate for surfaces which are not shadowed (mainly for large value of $h$ ) and make it smaller for shadowed one (for small value of $h)$. The diffusion is also affected by the shadowing in the same way.

A plane-wave analysis performed on equation (3) shows that the solutions are unstable for large enough wavelengths $\lambda$, i.e. $\lambda=2 \pi / k>\lambda^{*}=\nu \pi^{3} /(\alpha R)$, with $\alpha \sim 0.724$ and the growth rate $\sigma=k \pi\left(2 \alpha R-\nu \pi^{2} k\right)$. Then, starting from a flat substrate, the noise will trigger the instability and will drive the system into a strong non-linear regime. Figure 2 shows the evolution of the interface profile for different times for $D=1, \nu=1$ and $R=1$. It exhibits the desired columnar shape. This shape is characterised by flat column tops and vertical sides as compared to previous Monte-Carlo simulations and columnar growth experiments. The shadowed deposition favors the columnar growth and the anisotropic diffusion smoothes the top of the column very efficiently and leads to vertical sides. The competition between these two effects leads to a columnar regime as expected. Moreover, most of the columns formed at the beginning of the simulation are still present at the end. This is also the case for "Poisson/Wedding cakes" morphologies for which initial columns always remains [20] and for step meander process [17].

For numerical simulation, equation (3) is integrated using the following explicit scheme:

$$
\begin{aligned}
h_{i}^{n+1}= & h_{i}^{n}+\left(\Omega_{i}^{n}\right)^{2} \\
& {\left[\Delta t R \sqrt{1+\frac{\left(h_{i+1}^{n}-h_{i}^{n}\right)^{2}+\left(h_{i+1}^{n}-h_{i}^{n}\right)\left(h_{i}^{n}-h_{i-1}^{n}\right)+\left(h_{i}^{n}-h_{i-1}^{n}\right)^{2}}{3 \Delta x^{2}}}\right.}
\end{aligned}
$$




$$
\left.+\frac{\nu \Delta t}{\Delta x^{2}}\left(h_{i+1}^{n}-2 h_{i}^{n}+h_{i-1}^{n}\right)+\sqrt{\frac{2 D \Delta t}{\Delta x}} \varepsilon\right]
$$

with the notation $h_{i}^{n}=h(i \Delta x, n \Delta t) . \varepsilon$ is a random number picked with the uniform distribution between $[-1,1[$. To obtain a discrete form of the gradient term, we follow the scheme proposed by Lim et al. although their study strictly applied for the KPZ equation [19]. $\Omega(x,\{h\})$ is evaluated following reference [6].

The time evolution of the roughness $W$ of the interface is given figure 3 . It shows the existence of different regimes. The first one, for $t<1$ is driven by the fluctuations and $W$ scales as $t^{0.5}$. For the second one $(1<t<100)$, diffusion induced a relative reduction of the roughness which scales as $t^{0.4}$. Then, because of the shadowing instability described above, sharp canyons appear and the roughness quickly increases. Finally, after $t \sim 1000$, the columnar regime appears which leads to $W(t) \sim t$ as in the discrete model [14]. Even if $W(t)$ shows the same scaling as obtained in previous studies on continuous columnar growth model [14, 15, 16], the column shapes are rather different and are now in closer agreement with the MC models and more important with

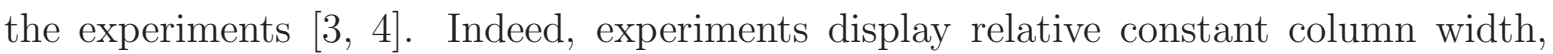
while MC simulations lead to a column width which is increasing with time. In that respect, our model (3) exhibits a better qualitative agreement with experiement than MC simulations.

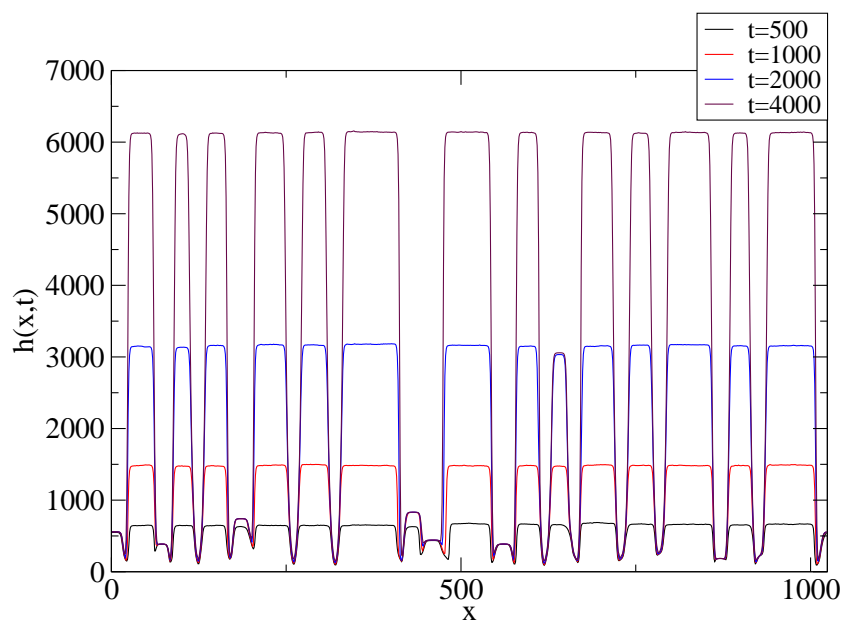

Figure 2. Continuous model. Snapshots of the interface given by the non-linear shadowing anisotropic diffusion model given by equation (3) at time $t=500,1000$, 2000 and 4000 . The numerical simulation was done with $\Delta t=0.01, \Delta x=1$ and the total suze of the system is $L=1024$.

We have presented the first continuous model, to our knowledge, that exhibits a columnar growth without the coarsening dynamics of the structures, in good agreement with experimental observations on sputtering deposition. For reproducing these wide flat columns with sharp edges, we have introduced an increase of the relaxation and 


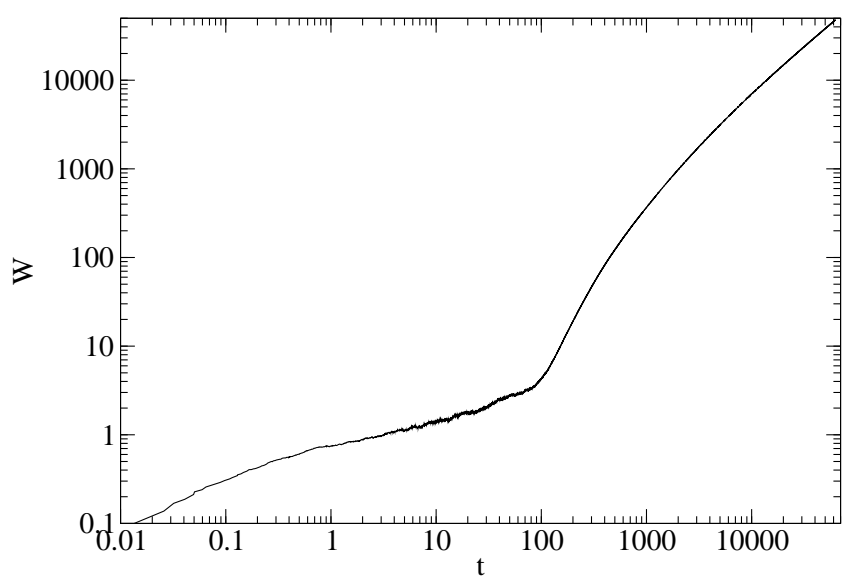

Figure 3. Continuous model. Roughness $W(t)$ as a function of time.

of the flux on the top of the column compared to the grooves. By sake of simplicity, we have considered a 1D surface and we have taken the same non local and nonlinear multiplicative factor $g(\Omega(x,\{h\}))$ for all the terms of the dynamics. Further on, we have considered a simple power law behaviour for this function $g(\Omega(x,\{h\}))=\Omega^{n}$. We argue that $n>1$ is needed to enhance the shadowing effects on the protuberances. We have tested numerically $n=2$ and $n=3$ with no loss of properties of the results. However, a better choice of the shadowing function $g$ should be obtained through further experimental comparisons. Similarly, different shadowing functions should be considered in the future for the diffusion term and the deposition term. Finally, this new continuous model, with $n=2$, considered as a minimal model, already correctly reproduces the formation of flat wide columns, with sharp edges and thin separating grooves, as usually encountered in sputtering deposition. Further works should perform such approaches to two dimensional surfaces in particular.

\section{Acknowledgments}

French Ministry of Research, Conseil Régional du Centre, Communauté d'Agglomération du Drouais, Conseil Général de l'Eure et Loir and CRT Plasma-Laser are acknowledged for a research grant. C.J. wants to thank the financial suport of the DGA.

\section{References}

[1] G. S. Bales and A. Zangwill, Macroscopic model for columnar growth of amorphous films by sputter deposition, J. Vac. Sci. Technol. A 9, pp 145-149 (1991).

[2] H. Rabat and P. Brault 2008 Plasma sputtering deposition of PEMFC porous carbon platinum, Fuel Cells, DOI: 1002/fuce.20070036 
[3] R. Messier, J. E. Yehoda Geometry of thin-film morphology, J Appl. Phys. 58 10, pp 3739-3746 (1985).

[4] A. G. Dirks, R. A. Wolters, A. E. M. De Veirman, Columnar Microstructures in MagnetronSputtered Refractory Metal Thin Films of Tungsten, Molybdenum and W-Ti-(N) Thin Solid Films 208 2, pp 181-188 (1992).

[5] G. S. Bales, R. Bruinsma, E. A. Eklund, R. P. U. Karunasiri, J. Rudnick, A. Zangwill, Growth and Erosion of Thin Solid Films, Science 249, pp 264-268 (1990).

[6] R.P.U. Karunasiri, R. Bruinsma, J. Rudnick, Thin film and the shadow instability, Phys. Rev. Lett. 62 7, pp 788-790 (1989).

[7] C. Roland, H. Guo, Interfacial Growth with a shadow instability Phys. Rev. Lett. 66, pp 2104-2107 (1991).

[8] P.I. Tamborenea and S. Das Sarma, Surface-diffusion-driven kinetic growth on one-dimensional substrates, Phys Rev E 48 4, pp 2575-2594 (1993).

[9] B. Meng, W.H. Weinberg, Dynamical Monte-Carlo studies of molecular beam epitaxial growth models : interfacial scaling and morphology, Surface Science 364, pp 151-163 (1996).

[10] K.A. Fichthorn, W.H. Weinberg, Theoretical foundations of dynamical Monte-Carlo simulations, J. Chem. Phys. 95 2, pp 1090-1096 (1991).

[11] S. Das Sarma, C. J. Lanczycki, R. Kotlyar, S. V. Ghaisas Scale invariance and dynamical correlations in growth models of molecular beam epitaxy, Phys. Rev. E 53, pp 359-389 (1996).

[12] M. Rost and J. Krug, Coarsening of surface structures in unstable epitaxial growth Phys. Rev. E 55, pp 3952-3957 (1997).

[13] J. G. Amar and F. Family, Numerical solution of a continuous equation for interface growth in 2+1 dimensions, Phys. Rev. A 41, pp 3399-3402 (1992).

[14] J.H. Yao, C. Roland, H. Guo, Interfacial dynamics with long-range screening, Phys. Rev. A 456 , pp 3903-3912 (1992).

[15] J.H. Yao, H. Guo, Shadowing in the three dimensions, Phys. Rev. E 47 2, pp 1007-1011 (1993).

[16] J. T. Drotar, Y.-P. Zhao, T.-M Lu, G.-C. Wang, Surface roughening growth and etching in 2+1 dimensions, Phys. Rev. B 62 3, pp 2118-2125 (2000).

[17] F. Gillet, O. Pierre-Louis, C. Misbah, Non-linear evolution of step meander during growth of a vicinal surface with no desorption, Eur. Phys. J. B 18, pp 519-534 (2000).

[18] M. Kardar, G. Parisi, Y.-C. Zhang, Dynamic scaling of growing interface, Phys. Rev. Letters 56 9, pp 889-892 (1986).

[19] C.-H. Lam and F.G. Shin, Anomaly in numerical integrations of the Kardar-Parisi-Zhang equation, Phys. Rev. E 576, pp 6506-6511 (1998).

[20] J. Krug, Origin of scale invariance in growth processes, Adv. Phys. 46, 1997, pp 139-282; J. Krug and M. Schimschak, Metastability of step flow growth in $1+1$ dimensions, J. Phys. I France 5, pp 1065-1086 (1995). 